Driven diffusion of vector fields

This article has been downloaded from IOPscience. Please scroll down to see the full text article.

1998 J. Phys. A: Math. Gen. 318355

(http://iopscience.iop.org/0305-4470/31/41/009)

View the table of contents for this issue, or go to the journal homepage for more

Download details:

IP Address: 147.96.14.16

The article was downloaded on 11/09/2013 at 16:40

Please note that terms and conditions apply. 


\title{
Driven diffusion of vector fields
}

\author{
S Marculescu $†$ and F Ruiz Ruiz $\ddagger \|$ \\ $\dagger$ Universität-GH Siegen, D-57068 Siegen, Germany \\ $\ddagger$ Departamento de Física Teórica I, Facultad de Ciencias Físicas, Universidad Complutense de \\ Madrid, E-28040 Madrid, Spain
}

Received 12 June 1998

\begin{abstract}
A model for the diffusion of vector fields driven by external forces is proposed. Using the renormalization group and the $\epsilon$-expansion, the dynamical critical properties of the model with Gaussian noise for dimensions below the critical dimension are investigated, and new transport universality classes are obtained.
\end{abstract}

Fourteen years ago it was suggested that driven diffusive systems may provide suitable models for fast ionic conductors and solid electrolytes [1]. This triggered interest in such systems, which over the years have become a very good scenario for investigating the properties of far-from-equilibrium steady states [2]. A simple microscopic realization of a driven diffusive system is an Ising lattice gas in contact with a thermal bath in which an external uniform driving force is introduced [1]. The usual hopping rate to nearest neighbours is modified through a bias in the rates for hopping along the direction of the external field. In a finite lattice with open boundary conditions, the gas evolves to a nonequilibrium steady state with a non-zero particle current. Due to the energy exchange of the system with the heat bath and the driving field, the system also has a steady energy current. So far driven diffusion of scalar quantities, like the excess of transported particles, has been considered. The purpose of this paper is to extend these considerations to vector quantities.

We begin by very briefly recalling the formulation for the standard case of a scalar quantity [3]. Space is taken to be $d$-dimensional and parametrized by coordinates $\left(x_{i}\right)=$ $\left(x_{\|}, \boldsymbol{x}_{\perp}\right)$, with $\|$ the direction of the driving force and $\perp$ the directions orthogonal to it. The deviation $s(x, t)$ of the density from its uniform average satisfies a continuity equation $\partial_{t} s+\partial_{i} J_{i}(s)=\eta$, with $J_{i}(s)$ the current generated by ordinary diffusion and the drift in the direction of the driving force, and $\eta(x, t)$ the noise accounting for the heat bath. The contribution of ordinary diffusion to the current is proportional to the gradient of $s$, while the drift in the $\|$-direction can be estimated, for small density fluctuations $s$, by expanding in powers of $s$ and retaining terms through order two. This gives the driven diffusion equation [3]

$$
\partial_{t} s-v \partial_{\|}^{2} s-v_{\perp} \Delta_{\perp} s+\frac{1}{2} \lambda \partial_{\|} s^{2}=\eta
$$

In the first papers [3] the noise was assumed to be the derivative of a random current, thus conserving the number of particles. In subsequent papers [4] Gaussian noise (describing

$\S$ E-mail: marculescu@physik.uni-siegen.d400.de

|| E-mail: ferruiz@eucmos.sim.ucm.es 
systems with random particle sources and drains and preserving the particle number only in the mean) was also considered. For both types of noise it was shown that driven diffusive systems have, for dimensions less than the critical dimension, infrared (IR) stable fixed points at which various quantities scale. Furthermore the corresponding critical exponents have been computed using the $\epsilon$-expansion $[3,4,6]$, the values obtained showing that density fluctuations spread faster than by diffusion.

It is worth noting that equation (1) also describes the toppling of grains of sand in a sandpile [6]. As a matter of fact, the system described by equation (1) with Gaussian noise was first considered as a model for the hydrodynamic regime of a running sandpile. However, one must mention that, after a shift, Gaussian noise has vanishing average and this requires particles to be randomly added and removed, whereas in the running sandpile particles are only added. In other words, Gaussian noise does not reproduce the feeding rate of the running sandpile [4]. It is also interesting to mention that for $d=1$ a field redefinition maps the driven diffusion equation (1) to the Kardar-Parisi-Zhang equation [5], which in turn is identical [2] to the Navier-Stokes equation. This is no longer true for $d \neq 1$, for which the three equations are different.

We now generalize driven diffusive systems to the case where the external forces acting on the system produce drift in a hyperplane of dimension $n$ parametrized by coordinates $x_{\alpha}(\alpha=1, \ldots, n)$. We do this by increasing the number of field variables from 1 to $n$, so that we have a vector $s_{\alpha}(x, t)$. The component $s_{\alpha}(x, t)$ describes the excess of grains transported along the direction $x_{\alpha}$. For every component $s_{\alpha}(x, t)$ we write a continuity equation $\partial_{t} s_{\alpha}+\partial_{i} J_{i \alpha}=\eta_{\alpha}$. Proceeding now as for the the standard case, we obtain

$\partial_{t} s_{\alpha}-\sigma \partial_{\beta} \partial_{\beta} s_{\alpha}-2 \sigma \xi \partial_{\alpha} \partial_{\beta} s_{\beta}-v_{\perp} \Delta_{\perp} s_{\alpha}+\frac{1}{2} \lambda \partial_{\alpha} s^{2}+\kappa \partial_{\beta}\left(n s_{\alpha} s_{\beta}-\delta_{\alpha \beta} s^{2}\right)=\eta_{\alpha}$

where we have used the notation $s^{2} \equiv s_{\alpha} s_{\alpha}$. This equation has two anisotropy coefficients, $\sigma$ and $\sigma \xi$, and two coupling constants, $\lambda$ and $\kappa$. For later convenience, we introduce the coefficient

$$
v \equiv \sigma(1+2 \xi) .
$$

Equation (2) makes sense for $1 \leqslant n \leqslant d$. If $n=1$, we recover the standard driven diffusion equation (1); if $n=d$, anisotropy is maximal and $v_{\perp}=0$. We wish to investigate the dynamical critical properties of driven diffusive systems based on this equation. In this paper, we concentrate on Gaussian noise

$$
\begin{aligned}
& \left\langle\eta_{\alpha}(x, t)\right\rangle=0 \\
& \left\langle\eta_{\alpha}(x, t) \eta_{\beta}\left(x^{\prime}, t^{\prime}\right)\right\rangle=2 \delta_{\alpha \beta} \delta^{(d)}\left(x-x^{\prime}\right) \delta\left(t-t^{\prime}\right)
\end{aligned}
$$

and use the renormalization group and the $\epsilon$-expansion to characterize the universality classes of the model to first order in $\epsilon$ for dimensions below the critical dimension. A similar programme can be carried for conserving noise.

We have already mentioned that equation (2) may be used to describe drift in a hyperplane. Scenarios in which this may become relevant are oil diffusion and diffusion in disorder media. More generally, equation (2) may be considered in connection with directed percolation [7]. We recall that in directed percolation, when the probability $p$ of jumping to the nearest neighbour along a preferred direction is set equal to a critical value $p_{*}$, a phase transition occurs. At this transition, the system shows scaling behaviour together with non-standard features, like, e.g., fractal dimensionality for the percolating cluster. One could think of the parameter $\xi$ as playing the role of the probability $p$, with the difference that $\xi$ is not a priori set equal to any critical value but enters the model as another parameter. The continuity equation (2) can also be thought of as describing the 
toppling of grains of sand in an $n$-dimensional sandpile, with $s_{\alpha}(x, t)$ the deviation in the direction $x_{\alpha}$ of the sandpile profile from its uniform average. Since in general $n \neq 1$, one may think of using (2) to describe the hydrodynamic regime of sandpiles with avalanches advancing anisotropically. Yet we must keep in mind that Gaussian noise does not describe the feeding rate of a sandpile. Other Langevin equations based on Gaussian noise describing surface sandpiles have been proposed in [10]. They differ from (2) in that they double the number of field variables but keep the number of transport directions equal to one. It is important to realize that equation (2) is not the spherical generalization of the ordinary driven diffusion equation (1) in which, while keeping the number of anisotropy directions equal to one, $s(x, t)$ becomes an $n$-vector field $(n=2 j+1)$ carrying an $n$-dimensional irreducible unitary representation of $O(3)$. For isotropic spherical generalizations of the Navier-Stokes equation and the Kardar-Parisi-Zhan equation, see [8] and [9].

To study the system (2) + (4), we use [11] and recast it as a stochastic quantum field theory model with classical action

$$
\begin{aligned}
S_{n}=\int \mathrm{d} t \mathrm{~d}^{d} x & \pi_{\alpha}\left[-\pi_{\alpha}+\partial_{t} s_{\alpha}-\sigma \partial_{\beta} \partial_{\beta} s_{\alpha}-2 \sigma \xi \partial_{\alpha} \partial_{\beta} s_{\beta}-v_{\perp} \Delta_{\perp} s_{\alpha}+\frac{1}{2} \lambda \partial_{\alpha} s^{2}\right. \\
& \left.+\kappa \partial_{\beta}\left(n s_{\alpha} s_{\beta}-\delta_{\alpha \beta} s^{2}\right)\right]
\end{aligned}
$$

where $\pi_{\alpha}(x, t)$ is the response field [12] to the assumed Gaussian noise. The action $S_{n}$ has the following symmetries.

(a) Translational invariance in all coordinates.

(b) $O(n) \otimes O(d-n)$ invariance with respect to spatial coordinates.

(c) Joint reflection of longitudinal coordinates, field variables and response fields.

(d) Scaling of longitudinal coordinates $\left(t, x_{\alpha}, x_{\perp}\right) \rightarrow\left(t, \varrho x_{\alpha}, x_{\perp}\right)$, with the anisotropy coefficients, coupling constants and fields transforming as $\left(\sigma, \xi, v_{\perp}\right) \rightarrow\left(\varrho^{2} \sigma, \xi, v_{\perp}\right)$, $(\lambda, \kappa) \rightarrow \varrho^{(n+2) / 2}(\lambda, \kappa)$ and $\left(s_{\alpha}, \pi_{\alpha}\right) \rightarrow \varrho^{-n / 2}\left(s_{\alpha}, \pi_{\alpha}\right)$. Since the parameter $\xi$ is scale invariant, we introduce the invariant coupling constants

$$
u \equiv \frac{1}{4 \pi} \frac{\lambda}{\sigma^{(n+2) / 4}} \quad v \equiv \frac{1}{4 \pi} \frac{\kappa}{\sigma^{(n+2) / 4}} .
$$

In addition, if $n=1$, the action is invariant under Galilei transformations $[4,6]$

$$
\begin{aligned}
& s(x, t) \rightarrow s\left(x_{\perp}, x_{\|}-\lambda a t, t\right)+a \\
& \pi(x, t) \rightarrow \pi\left(x_{\perp}, x_{\|}-\lambda a t, t\right) .
\end{aligned}
$$

We note that this symmetry does not generalize to $n>1$. The reason is that Galilei's invariance requires a constant velocity vector and this is only possible for $n=1$, since in this case there is only one preferred direction. In other words, it is precisely the passage from $n=1$ to $n \neq 1$ what destroys the Galilei symmetry.

The critical dimension of the model is $d_{c}=4$. For $d=4$ the model is ultraviolet renormalizable by power counting, the only primitively divergent one-particle irreducible Green functions being the self-energy $\left\langle\pi_{\alpha}(p) s_{\beta}(-p)\right\rangle$ and the vertex $\left\langle\pi_{\alpha}(p) s_{\beta}(q) s_{\gamma}(-p-\right.$ $q)\rangle$, which have linear and logarithmic degrees of divergence respectively. Using dimensional regularization [13], we obtain the following one-loop renormalization group equations:

$$
\begin{aligned}
& \beta_{u} \equiv \frac{\mathrm{d} u}{\mathrm{~d} \ln \mu}=-u\left(\frac{\epsilon}{2}+\mathcal{H}+n \mathcal{L}+\frac{n+2}{4} \mathcal{F}\right) \\
& \beta_{v} \equiv \frac{\mathrm{d} v}{\mathrm{~d} \ln \mu}=-v\left(\frac{\epsilon}{2}+\mathcal{H}+\frac{n+2}{4} \mathcal{F}\right)
\end{aligned}
$$




$$
\begin{aligned}
\beta_{\xi} & \equiv \frac{\mathrm{d} \xi}{\mathrm{d} \ln \mu}=\xi(\mathcal{G}-\mathcal{F}) \\
\zeta_{\sigma} & \equiv \frac{\mathrm{d} \ln \sigma}{\mathrm{d} \ln \mu}=\mathcal{F} \\
\zeta_{\nu} & \equiv \frac{\mathrm{d} \ln \nu}{\mathrm{d} \ln \mu}=\frac{\mathcal{F}+2 \xi \mathcal{G}}{1+2 \xi}
\end{aligned}
$$

where $\mu$ is the dimensional regularization mass scale, $\epsilon=4-d$ is the deviation from the critical dimension and $\mathcal{F}, \mathcal{G}, \mathcal{H}$ and $\mathcal{L}$ are functions of $u, v$ and $\xi$ given by

$n(n+2) \mathcal{X}(u, v, \xi)=u^{2} x_{u u}(\xi)+u v x_{u v}(\xi)+v^{2} x_{v v}(\xi) \quad \mathcal{X} \equiv \mathcal{F}, \mathcal{G}, \mathcal{H}, \mathcal{L}$.

For completeness we write the expressions of $x_{u u}, x_{u v}$ and $x_{v v}$ :

$$
\begin{aligned}
& f_{u u}=0 \\
& f_{u v}=\left[\frac{n^{2}}{\xi}-n(n+1)(n-2)\right] a_{1}-n^{2}\left(\frac{1}{\xi}+2\right) a_{2}-\frac{n}{2}(n+4) a_{3} \\
& f_{v v}=\frac{n^{2}}{2}\left(-2 n^{2}+n+4\right)-2 n\left(\frac{n}{\xi}+n+2\right) a_{1}+n^{2}\left(\frac{n+2}{\xi}+4\right) a_{2}-n^{2}\left(\frac{n}{\xi}-n+2\right) a_{3} \\
& -n\left(n^{2}-n-4\right) a_{4} \\
& g_{u u}=-\frac{n+2}{2 \xi}\left[(n-1) a_{1}+\left(1-\frac{n}{4}\right) a_{4}\right] \\
& g_{u v}=\frac{n}{2 \xi}\left[-\frac{1}{4}(n-1)(n+2)(3 n+4)+\left(\frac{n-2}{\xi}-n^{2}+5 n+2-\frac{8}{n}\right) a_{1}\right. \\
& \left.-(n-2)\left(\frac{1}{\xi}+2\right) a_{2}+(n-1)(n+2) a_{3}-\left(\frac{3}{2} n^{2}+4 n-\frac{8}{n}\right) a_{4}\right] \\
& g_{v v}=\frac{n-2}{2 n \xi} f_{v v} \\
& h_{u u}=\frac{2}{\xi}\left[(n+1) a_{1}-n a_{2}-a_{3}\right] \\
& h_{u v}=\frac{2 n}{\xi}\left[n^{2}-2-(n+1)\left(n-2+\frac{4}{n}\right) a_{1}-2(n-2) a_{2}+\left(n-2+\frac{4}{n}\right) a_{3}+2 a_{4}\right] \\
& h_{v v}=-\frac{n}{\xi}\left[4\left(n^{2}-2\right)+\left(n^{3}-4 n^{2}+2 n-\frac{8}{n}\right) a_{1}+2(n-2)^{2} a_{2}\right. \\
& \left.-\left(n^{3}+2 n^{2}-6 n+8-\frac{8}{n}\right) a_{3}+8 a_{4}\right] \\
& \ell_{u u}=\frac{1}{\xi}\left[-(n+3) a_{1}+2 a_{2}+(n+1) a_{3}\right] \\
& \ell_{u v}=\frac{2 n}{\xi}\left[1+\frac{6}{n} a_{1}+\frac{2(n-2)}{n} a_{2}+\left(n-2-\frac{2}{n}\right) a_{3}-(n+1) a_{4}\right] \\
& \ell_{v v}=-\frac{n}{\xi}\left[4+\left(n-4+\frac{12}{n}\right) a_{1}-\frac{2(n-2)^{2}}{n} a_{2}+\left(5 n-4-\frac{4}{n}\right) a_{3}-4(n+1) a_{4}\right]
\end{aligned}
$$


with $a_{1}, a_{2}, a_{3}$ and $a_{4}$ taking the form

$$
\begin{array}{ll}
a_{1}=a_{2} F\left(\frac{n}{2}, 1 ; 2 ; \frac{\xi}{1+\xi}\right) & a_{2}=\frac{1}{(1+\xi)^{n / 2}} \\
a_{3}=a_{4} F\left(\frac{n}{2}, 1 ; 2 ;-\frac{\xi}{1+\xi}\right) & a_{4}=\frac{1}{(1+2 \xi)^{n / 2}}
\end{array}
$$

and $F(a, b ; c ; z)$ being the hypergeometric function. For $n=1$ there is no $\kappa$-term in $S_{n}$, so that $\kappa=0$ and hence $v=0$. From this, and using $n=1$, it follows that $\mathcal{F}=\mathcal{H}+n \mathcal{L}=0$. Thus $\beta_{u}=\epsilon / 2$ and, as a result, $\beta_{u}=0$ at the critical dimension, implying no ultraviolet renormalization of $\lambda$. This lack of renormalization holds to all orders in perturbation theory and follows [6] from the non-anomalous character of the Galilei symmetry of the $n=1$ model mentioned above. For an $n=1$ driven diffusive system which is classically Galilei invariant but has a quantum mechanical anomaly, see [14].

To characterize the critical behaviour of the model for $d \leqslant d_{c}$, we solve the renormalization group equations in the neighbourhood of all IR attractive fixed points $\left(u_{*}, v_{*}, \xi_{*}\right)$. Equations (6)-(8) form a system of differential equations describing the dependence of $u, v$ and $\xi$ on $\mu$. Upon substitution in (9), (10), its solution near an IR attractive fixed point gives the $\mu$-dependence of the anisotropy coefficients $\sigma$ and $v$ near the fixed point. Indeed, in the neighbourhood of a fixed point, the solutions to (9), (10) $\operatorname{read} \sigma \propto \mu^{\zeta_{\sigma *}}$ and $\nu \propto \mu^{\zeta_{\nu *}}$, with

$$
\left.\left.\zeta_{\sigma *} \equiv \mathcal{F}\right|_{*} \quad \zeta_{\nu *} \equiv \frac{\mathcal{F}+2 \xi \mathcal{G}}{1+2 \xi}\right|_{*}
$$

To have a signal for criticality, at least one of the two exponents $\zeta_{\sigma *}$ and $\zeta_{\nu *}$ must be negative, since $\sigma$ or $v$ will then diverge as $\mu \rightarrow 0$. To find the IR attractive fixed points with diverging length scales of the model for $d \leqslant d_{c}$, we therefore proceed in two steps:

Step 1. We first find all fixed points by solving the equations $\beta_{u}=\beta_{v}=\beta_{\xi}=0$ and retain those which are IR attractive. We remind that for a fixed point to be IR attractive, the matrix of derivatives

$$
\left(\begin{array}{ccc}
\beta_{u u} & \beta_{u v} & \beta_{u \xi} \\
\beta_{v u} & \beta_{v v} & \beta_{v \xi} \\
\beta_{\xi u} & \beta_{\xi v} & \beta_{\xi \xi}
\end{array}\right) \quad \beta_{x y}=\left.\frac{\partial \beta_{x}}{\partial y}\right|_{*}
$$

which results from linearizing equations (6)-(8) about the fixed point must have eigenvalues with strictly positive real parts. The case $\xi_{*} \rightarrow \infty$ must be treated separately, since then there is no renormalization of $\xi$, hence no equation (8). In this case, fixed points are solutions of $\beta_{u}=\beta_{v}=0$ and the matrix of derivatives of the beta functions is the upper left-hand $2 \times 2$ sub-matrix of (11).

Step 2. Next we calculate $\zeta_{\sigma *}$ and $\zeta_{\nu *}$ for all the IR attractive fixed points found in step 1 and keep those values which are negative. For $\xi_{*}$ finite, the condition $\beta_{\xi}=0$ and equation (8) imply that the two critical exponents take the same value $\zeta_{\sigma *}=\zeta_{\nu *}$. For $\xi_{*} \rightarrow \infty$, however, the condition $\beta_{\xi}=0$ is no longer required and $\zeta_{\sigma *}$ and $\zeta_{\nu *}$ may take different values.

Although $n$ has been assumed to take integer values in the range 1 to 4 , the results can be analytically continued in $n$. In fact, analytic continuation gives well-defined beta functions for exotic values of $n$, like for example $n \rightarrow 0$ or $n \rightarrow-2$. In what follows we take $n$ to be a real variable.

$\dagger$ This also follows from the definitions of $v, \zeta_{\sigma}$ and $\zeta_{v}$ given in equations (3), (9) and (10). 
In step 1 of this procedure, one must keep in mind that the values $u_{*}$ and $v_{*}$ of the couplings $u$ and $v$ at a fixed point are not physically meaningful. Hence redefining $\bar{u}=\chi u$ and $\bar{v}=\psi v$, with $\chi$ and $\psi$ functions of $\xi$, does not change the physics. Such a redefinition may be used when one or both of $u_{*}$ and $v_{*}$ go to infinity to move the fixed point to $\bar{u}_{*}$ and $\bar{v}_{*}$ finite. The renormalization group equations for $\bar{u}$ and $\bar{v}$ can easily be obtained from (6)-(8) and involve the functions $\chi$ and $\psi$. The functions $\chi$ and $\psi$ must be found such that the IR attractive fixed points of the new renormalization group equations have $\bar{u}_{*}$ and $\bar{v}_{*}$ finite. This introduces two new unknowns, $\chi$ and $\psi$, which, together with the complexity of the functions $\mathcal{F}, \mathcal{G}, \mathcal{H}$ and $\mathcal{L}$, makes it infeasible to carry the above programme analytically keeping $\xi$ and $n$ arbitrary. We have thus restricted ourselves to some values of $n$ and $\xi$ of interest. In particular, we have treated the cases

(i) $n=-2,0,1,2,3,4$ with arbitrary $\left(u_{*}, v_{*}, \xi_{*}\right)$;

(ii) $\xi_{*}=0,(n-2) / 2 n, \infty$ with arbitrary $\left(n, u_{*}, v_{*}\right)$;

allowing in both instances for redefinitions of $u$ and $v$ as described above. The values $\xi_{*}=0$ and $\xi_{*}=\infty$ considered in case (ii) correspond respectively to $\pi_{\alpha} \partial^{2} s_{\alpha}$ and $\pi_{\alpha} \partial_{\alpha} \partial_{\beta} s_{\beta}$ being the dominant kinetic anisotropic term in the classical action $S_{n}$. The idea of looking for critical points at $\xi_{*}=(n-2) / 2 n$ stems from the simple relation between $f_{v v}$ and $g_{v v}$ for arbitrary $n$, namely $2 n \xi g_{v v}=(n-2) f_{v v}$. Because of this relation, the equation $\beta_{\xi}=0$ simplifies considerably if $\xi_{*}=(n-2) / 2 n$, thus making it possible to find IR attractive fixed points with finite $\xi_{*}$. Another motivation for considering $\xi_{*}=(n-2) / 2 n$ is to investigate whether the passage from $n \neq 1$ to $n=1$ is smooth, since the case $n=1$ can be recovered from $\xi_{*}=-\frac{1}{2}$ and $\sigma \rightarrow \infty$.

Table 1. Universality classes for $n=3,4$.

\begin{tabular}{llrl}
\hline$n$ & $\xi_{*}$ & $\zeta_{\nu *}$ & $\zeta_{\sigma *}$ \\
\hline 3 & $1 / 6$ & $-0.1257 \epsilon$ & $-0.1257 \epsilon$ \\
& $\infty$ & Positive & $-0.2848 \epsilon$ \\
& $\infty$ & $-0.1068 \epsilon$ & $-0.4726 \epsilon$ \\
4 & $1 / 4$ & $-158 \epsilon / 629$ & $-158 \epsilon / 629$ \\
& $\infty$ & Positive & $-0.3731 \epsilon$ \\
& $\infty$ & $-0.3731 \epsilon$ & $-0.3526 \epsilon$ \\
\hline
\end{tabular}

The results that we have obtained for (i) and (ii) are as follows (we provide the values of the physical quantities $n, \zeta_{\nu *}$ and $\zeta_{\sigma *}$, which characterize the universality classes). For case (i) there are no IR attractive fixed points with diverging length scales if $n=-2,0,2$. If $n=1$, the results of [6] are recovered. And if $n=3,4$, we obtain the universality classes in table 1 . For case (ii) we obtain the universality classes:

- $\xi_{*}=(n-2) / 2 n$, with $n>2$ and critical exponents

$$
\zeta_{\sigma *}=\zeta_{v *}=-\left.\frac{2 f_{v v} \epsilon}{(n+2) f_{v v}+4 h_{v v}}\right|_{\xi=(n-2) / 2 n} .
$$

Note that the critical exponent is a complicated but well-defined function of $n$.

- $\xi_{*}=\infty$, with $n=n_{0} \equiv(1+\sqrt{33}) / 4$ and critical exponents

$$
\zeta_{\nu *}=0 \quad \zeta_{\sigma *}=-\epsilon .
$$


- $\xi_{*}=\infty$, with $n>2$ and critical exponents

$$
\begin{aligned}
& \zeta_{\sigma *}^{ \pm}=\frac{1}{2} n b_{1} \epsilon \frac{2(n+1) X_{ \pm}+n b_{3}}{X_{ \pm}\left(b_{1} b_{5}-b_{2} b_{4}\right)+b_{1} b_{6}-b_{3} b_{4}} \\
& \zeta_{\nu *}^{ \pm}=\frac{1}{16}(n-1) \epsilon \frac{X_{ \pm}\left[n(3 n+4)(n-2) b_{1}-8 b_{2}\right]-8 b_{3}}{X_{ \pm}\left(b_{1} b_{5}-b_{2} b_{4}\right)+b_{1} b_{6}-b_{3} b_{4}}
\end{aligned}
$$

where

$$
\begin{aligned}
& b_{1}=-\frac{1}{n-2}\left(3 n^{2}+7 n-2\right) \quad b_{4}=-\frac{1}{n-2}\left(n^{2}-3 n-6\right) \\
& b_{2}=-\frac{1}{8} n(3 n+1)\left(n^{2}+2 n-12\right) \quad b_{5}=\frac{1}{2} n\left(3 n^{2}-3 n-10\right) \\
& b_{3}=\frac{1}{2}\left(2 n^{2}-n-4\right) \quad b_{6}=-\frac{1}{8} n^{2}(n+2)\left(2 n^{2}-n-4\right)
\end{aligned}
$$

and

$$
X_{ \pm}=\frac{1}{2 b_{1}}\left(-b_{2} \pm \sqrt{b_{2}^{2}-4 b_{1} b_{3}}\right) .
$$

The exponents $\zeta_{\nu *}^{-}$and $\zeta_{\sigma *}^{ \pm}$are negative for all $2<n \leqslant 4$, whereas $\zeta_{v *}^{+}$is negative only for $2<n<2.0060$. It is worth noting that the universality classes for $n=-2,0,2,3,4$ are particular cases of equations (12)-(15).

The anomalous dimensions $\zeta_{\nu *}$ and $\zeta_{\sigma *}$ found above are related to power laws in physical correlation functions and therefore should be directly measurable. In accordance with the continuity equation (2) and owing to the vector character of $s_{\alpha}$, physical correlators are expectation values of products of tensor currents

$$
J_{\alpha \beta}=-\sigma \partial_{\alpha} s_{\beta}-2 \sigma \xi \partial_{\beta} s_{\alpha}+\frac{1}{2} \lambda \delta_{\alpha \beta} s^{2}+\kappa\left(n s_{\alpha} s_{\beta}-\delta_{\alpha \beta} s^{2}\right) .
$$

Since $J_{\alpha \beta}$ contains linear and quadratic terms in $s_{\alpha}$, the computation of physical correlators involves renormalization of composite operators. For $n=1$, due to the non-renormalization of the coupling constant, renormalization of composite operators can be fully eliminated by considering correlators quadratic in the current [4]. For $n \neq 1$, since the coupling constants do renormalize, correlators cubic in $J_{\alpha \beta}$ must also be considered. The analysis of physical correlators and their relation to the critical exponents $\zeta_{\nu *}$ and $\zeta_{\sigma *}$ is in progress and will be presented elsewhere.

We conclude with a few comments.

Comment 1 . The anisotropy dimension $n$ at which the critical exponents $\zeta_{\nu *}$ and $\zeta_{\sigma *}$ occur is in general non-integer. It is particularly noticeable that for $\bar{u}_{*}^{2}=0, \bar{v}_{*}^{2}$ finite and $\xi_{*}=\infty$, the only IR attractive fixed point with diverging length scales is that given in (13) and occurs at $n=(1+\sqrt{33}) / 4$. With the caution due to the one-loop nature of our results, this suggests a fractal behaviour of the model. The similarity in some regards of our model to directed percolation may provide some insight in understanding these points.

Comment 2. The fixed points associated with the critical exponents in (12), (13) have either $\bar{u}_{*}=0$ or $\bar{v}_{*}=0$, while the fixed points associated with the critical exponents in (14), (15) have $\bar{u}_{*}, \bar{v}_{*} \neq 0$. We have not been able to find IR attractive fixed points with all three parameters $\bar{u}_{*}, \bar{v}_{*}$ and $\xi_{*}$ finite and non-zero. Our results show that if such a point exists to first order in $\epsilon$, it has fractal dimension. 
Comment 3. Equations (14), (15) give for $n>2$ two sets of critical exponents at $\xi_{*}=\infty$ which are associated with two different attractive IR fixed points. This is so since $\bar{u}_{*}^{2}$ and $\bar{v}_{*}^{2}$ are two-valued functions of $\xi$ for $\xi \rightarrow \infty$ and $\bar{u}_{*}, \bar{v}_{*} \neq 0$. Depending on the configuration of all fixed points, the system will flow in parameter space to one or another IR fixed point and will reach one or another set of critical exponents.

Comment 4. If we take $\lambda=0$ in $S_{n}$, we find universality classes at $\xi_{*}=\infty$ for $n>n_{0}$, with critical exponents $\zeta_{\nu *}=0$ and $\zeta_{\sigma *}=-2 \epsilon /(n+2)$. On the other hand, if we set $\kappa=0$, we obtain universality classes at $\xi_{*}=\infty$ for $n>2$, with critical exponents $\zeta_{\nu *}=0$ and $\zeta_{\sigma *}=-\epsilon / 6$. Note however that, despite the simplicity and beauty of these results, for $\lambda=0$ or $\kappa=0$ perturbation theory may at higher loops generate divergent relevant operators which are not in the classical action $S_{n}$. For this reason, we have considered the more general case of two coupling constants.

\section{Acknowledgments}

The authors are grateful to Bo Zheng for many clarifying discussions. FRR is grateful to the Institute for Theoretical Physics in Heidelberg for its hospitality and to the Alexander von Humboldt Foundation for support through a Research Fellowship.

\section{References}

[1] Katz S, Lebowitz J L and Spohn H 1983 Phys. Rev. B28 1655 Katz S, Lebowitz J L and Spohn H 1984 J. Stat. Phys. 34497

[2] Schmittmann B and Zia R K P 1995 Statistical Mechanics of Driven Diffusive Systems (Phase Transitions and Critical Phenomena 17) ed C Domb and J L Lebowitz (New York: Academic)

[3] Janssen H K and Schmittmann B 1986 Z. Phys. B 63517 Janssen H K and Schmittmann B 1986 Z. Phys. B 64503

[4] Becker V and Janssen H K 1994 Phys. Rev. E 501114

[5] Kardar M, Parisi G and Zhang Y C 1986 Phys. Rev. Lett. 56889

[6] Hwa T and Kardar M 1989 Phys. Rev. Lett. 621813 Hwa T and Kardar M 1992 Phys. Rev. A 457002

[7] Stauffer D and Aharony A 1994 Percolation Theory (London: Taylor and Francis)

[8] You C Y and Weichman P 1993 Phys. Rev. Lett. 701101

[9] Doherty J P 1994 Phys. Rev. Lett. 722041

[10] Bouchard J P, Cates M E, Ravi Prakash J and Edwards S F 1994 J. Physique I 41383 Mehta A, Luck J M and Needs R J 1996 Phys. Rev. E 5392

Biswas P, Majumdar A, Mehta A and Bhattacharjee J K 1997 Some properties of a class of coupled Langevin equations, cond-mat/9708155

[11] Janssen H K 1976 Z. Phys. B 23377 De Dominicis C 1976 J. Physique Coll. 37 C-247

[12] Martin P C, Siggia E D and Rose H H 1973 Phys. Rev. A 8423

[13] 't Hooft G and Veltman M 1972 Nucl. Phys. B 44189

[14] Janssen H K 1997 Phys. Rev. Lett. 781082 